\title{
Why we Need a Heated Chamber for 3D Printing with 'High Performance' Polymers?
}

\author{
Evgeny Boytsov ${ }^{1, a}$, Sergei Blaginin ${ }^{1, b}$ and Alexey Sinkov²,c* \\ ${ }^{1}$ LLC "VOLTEHNO", 404106, Volzhsky, Prospekt Lenina st., 308k, Volgograd region, Russia \\ ${ }^{2}$ Volzhsky Polytechnic Institute (branch) of VolgSTU, 404121, Volzhsky, Engels st., 42a., \\ Volgograd region, Russia \\ adiamagnetik@inbox.ru, bdemilano@mail.ru, csinkov73@mail.ru
}

Keywords: High Performance Polymers, High-Temperature Polymers, 3D Printer, Heated Chamber, Glass Transition Temperature, PEEK, FDM, FFF, Filament, Viscosity, Shrinkage Rate, Warpage, Annealing, Extrusion

Abstract. This paper is dedicated to the features of the processes of 3D printing of polymers with a high melting point such as PEEK, CarbonPEEK, ULTEM, PPSU using FDM (FFF) technology. The results of 3D printing with high-performance polymers using different conditions of the heated chamber are presented. Conclusions about the advantages of a heated chamber are made.

\section{Introduction}

Additive manufacturing technologies, in particular FDM or FFF 3D printing technologies, have become widespread and are of great interest in industry, medicine, science and other fields of activity due to their potential to simplify and reduce the cost of complex industries and the attractiveness of specialized research.

FDM technology implies the creation of 3D objects by applying successive layers of material that follow the contours of the digital model (Fig. 1). Typically, thermoplastic polymers supplied in the form of filaments or rods are used as printing materials.

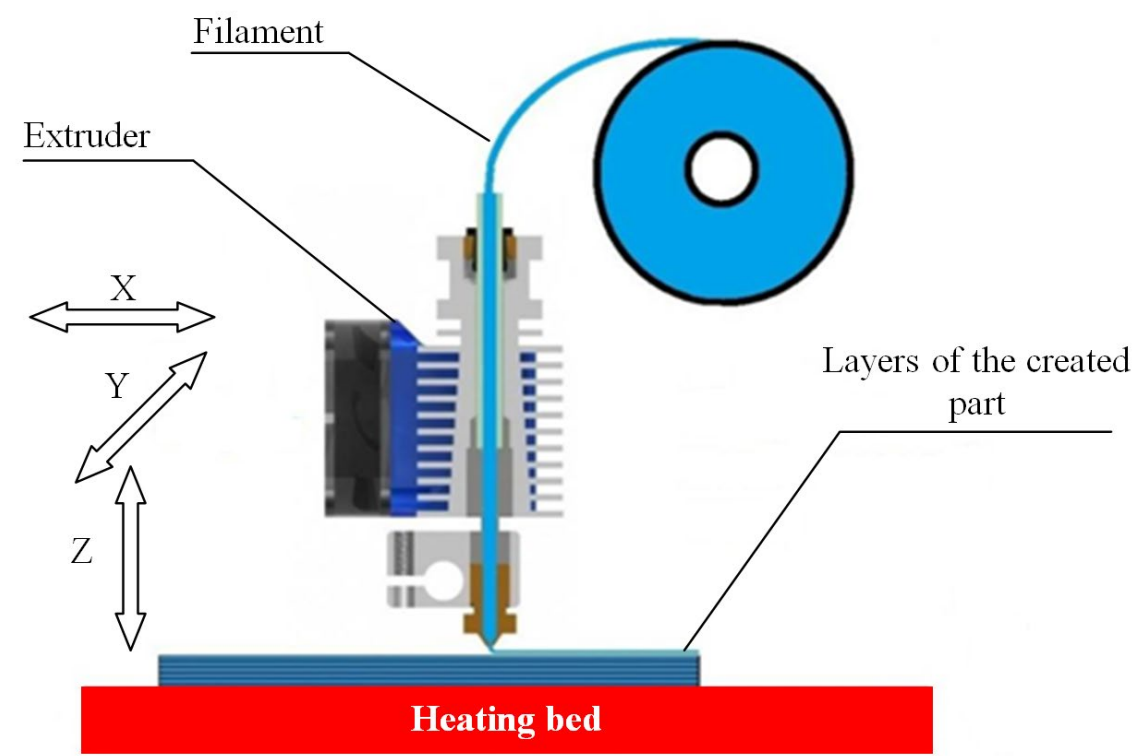

Fig. 1. FDM printing scheme. X, Y, Z-directions of movement of the extruder. 
Currently, the manufacture of parts and devices using FDM technologies has reached a new level in connection with the use of high-performance polymers such as PEEK [1], CarbonPEEK [2], ULTEM1010, ULTEM9085, PPSU, etc. as filaments (fig. 2). Products made from such materials have a thermal resistance above $100^{\circ} \mathrm{C}$, have good chemical resistance to many aggressive environment, and in terms of strength characteristics are approach to the properties of some metals $[3,4]$.

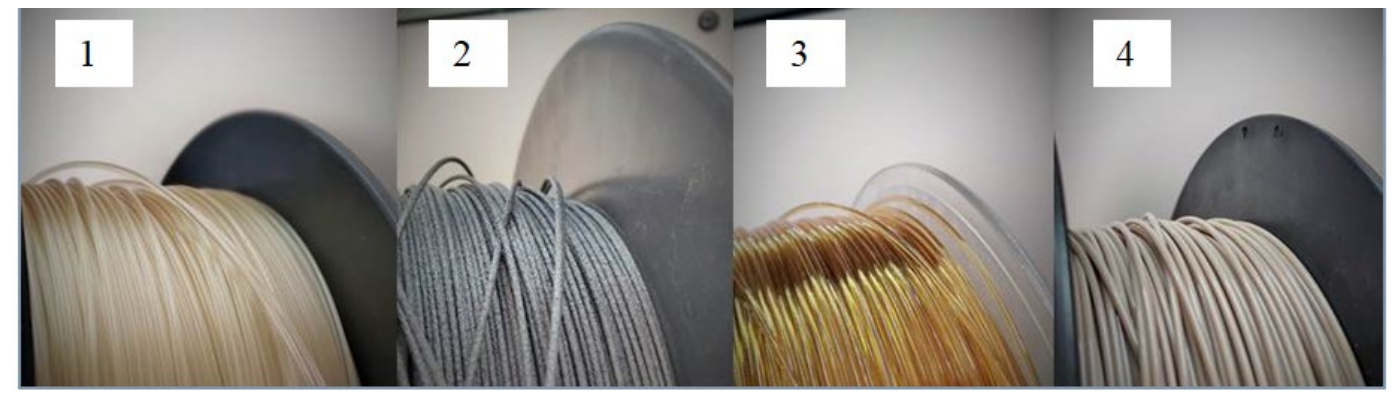

Fig. 2. Filaments of high-performance polymers: 1 - PPSU; 2 - CarbonPEEK; 3 - ULTEM; 4 PEEK

For example, Airbus (a European manufacturer of aviation equipment), under the program for the development of plane of the A-350 type, has mastered the manufacture of more than 3,000 parts from high-performance polymers using FDM technologies. These are elements of the interior of the passenger compartment, fasteners, fittings, air ducts and supporting unloaded elements.

It is known that 3D printing with high-performance polymers is associated with some technological difficulties. To overcome such difficulties, a 3D printer must have certain characteristics. For a better understanding of what characteristics a 3D printer that prints with highperformance polymers should have, we will consider the main aspects of 3D printing technology with such materials.

When using the FDM technology, the filament is first heated above the melting temperature and the polymer passes from a solid to a liquid state. Liquid polymer is extruded through a thin channel - a nozzle. After extrusion, the polymer is cooled in the environment and a reverse transition from liquid to solid occurs. Thus, in the process of 3D printing, there are two phase transformations of the polymer: from solid to liquid and from liquid to solid.

High-performance polymers have a very high melting point, and the extrusion process is carried out at even higher temperatures. For example, PEEK polymer is extruded at temperatures around $450^{\circ} \mathrm{C}$. When such a polymer is extruded into the environment at room temperature, a large temperature difference (temperature gradient) is obtained between the plastic and the environment. The high temperature gradient between the liquid polymer and the environment leads to intensive cooling of the surface layers of the product, during which there is a rapid transition from the liquid phase to the solid phase.

During rapid polymerization, the molecules of the material remain in a disordered position. This phenomenon causes increased stresses in the surface layers of the material. On finished products, this manifests itself in the form of delamination or cracks between the printed layers, a strong deviation from the initial dimensions and shape of the virtual model, high surface roughness and other negative effects are observed. As a result, such products have reduced thermal and mechanical properties. 
High-performance polymers have increased viscosities resulting in high shrinkage rates on rapid cooling. It also makes it difficult to obtain the desired dimensional tolerances on the manufactured parts.

Thus, the presence of a high temperature difference between the extruded polymer and the environment leads to a number of negative effects.

To overcome or minimize these problems, several functions can be implemented to ensure that high-performance polymers are 3D printed with the best possible quality.

There are currently only a few companies capable of printing high-performance polymers with high precision and high quality. The reason for this lies in the knowledge of the chemical and physical features of these high-performance materials and the ability to translate these features into the designs and settings of 3D printers, allowing to provide the necessary conditions for the production of quality products.

\section{Effect of polymer viscosity}

When extruded, the filament melts and becomes a liquid, so viscosity will affect its behavior.

The viscosity of a liquid polymer is the amount of resistance to flow due to internal friction between adjacent layers of liquid sliding against each other.

In a traditional extruder design, the flow of liquid polymer exiting the extruder nozzle is limited by the friction generated on the inner surface of the channel. This leads to a decrease in the flow area of the nozzle channel and periodic clogging of the extruder. In addition, during the passage of the melt inside the nozzle channel, air enters together with the polymer, creating air cavities between the polymer layers. This causes a sharp deterioration in the mechanical properties of the part being created. As the diameter of the extruder nozzle decreases and the print speed increases, the negative effect of polymer viscosity affects print quality more intensively.

To solve the problems arising from the negative influence of polymer viscosity, a new extrusion system was needed. The research department of VOLTEKHNO LLC has developed its own design of the extruder (Fig. 3), which is completed with the 3DLIFE THERMO printer [5].

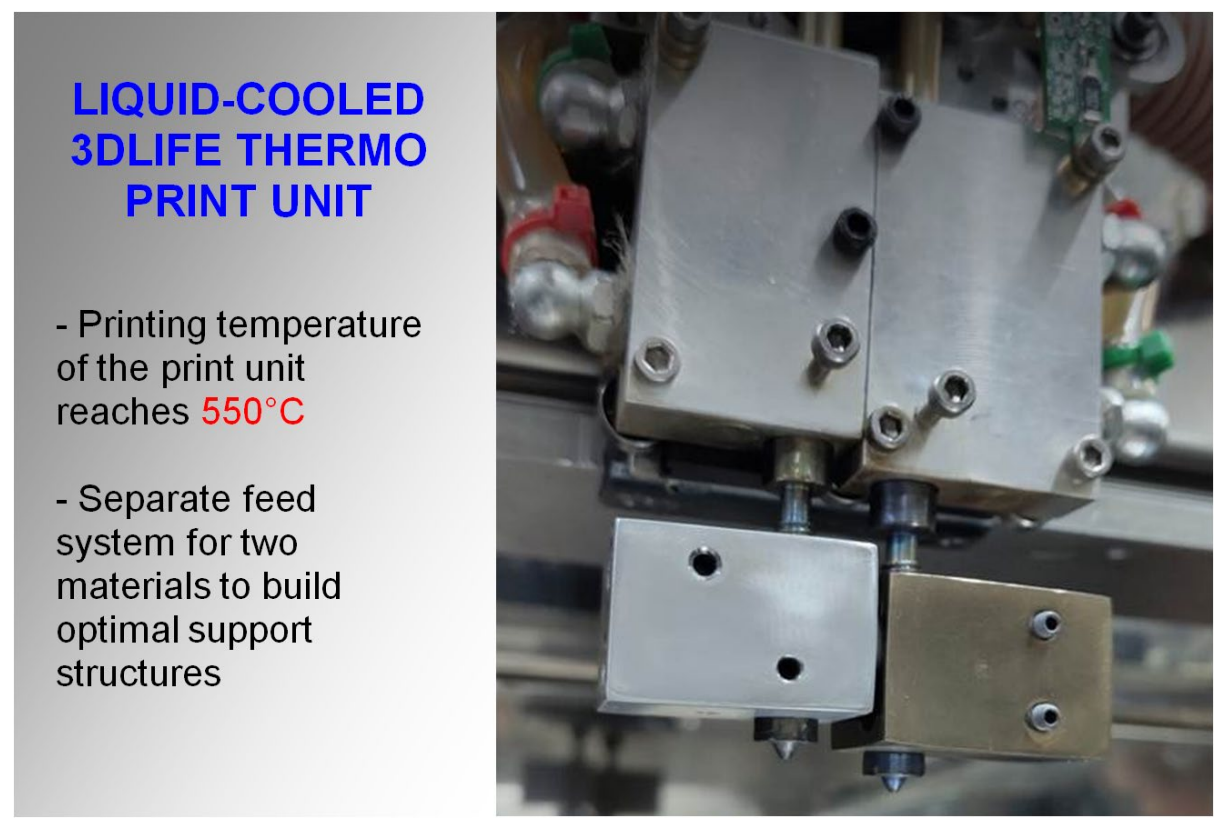

Fig. 3. Liquid-cooled 3DLIFE THERMO print unit. 
The use of a friction-reducing ceramic inner bore facilitates the extrusion of highly viscous polymers. The special profiling of the inner channel allows for an acceleration of the polymer flow. The narrowing of the extrusion section leads to an increase in the shear stress acting on the polymer melt, which reduces its viscosity. Equipping the extruder with liquid cooling is very important for the thermoregulation of individual parts of the extruder, especially when the printing temperature reaches $450^{\circ} \mathrm{C}$.

The technical solutions used in the design of the extruder allow creating an optimized polymer flow, reducing the negative phenomena of shrinkage, ensuring low dimensional tolerances for the finished product and maintaining the chemical-physical properties of the material [6].

\section{Shrinkage rate and warpage of polymers}

Shrinkage rate is a property of polymeric materials that determines the accuracy and size of parts, expressed as a percentage. This parameter is defined as the rate of volumetric contraction during the transition of the polymer from the liquid phase of the melt to the solid phase of the cooled state.

When the polymer is extruded into an environment with room temperature $\left(20-30^{\circ} \mathrm{C}\right)$, there is a large temperature difference between the melt and the medium, which leads to a high cooling rate of the polymer. The cooling rate affects the processes occurring during the crystallization of the material, namely: the higher the printing temperature, the higher the cooling rate and the higher the shrinkage rate.

The shrinkage rate of polymers depends on the material and varies for most thermo polymers in the range from 0.2 to $1 \%$. Shrinkage of high-temperature polymers can be up to $2 \%$, which causes certain difficulties in printing.

Parts printed with highly shrinkable resins often have a warping effect. The warping effect is manifested in the detachment of plastic layers from the surface of the heated table on which the product is printed. The warping effect is especially pronounced at the edges of the first layer around the perimeter of the part being created. This is due to the fact that when the first layer is printed, the melt is extruded onto the table surface, and upon cooling, the polymer adheres to the table material. Thus, during the phase transition of a liquid into a solid state, adhesion of dissimilar bodies occurs: liquid and solid due to the forces of interfacial adhesion. Further cooling of the polymer is accompanied by shrinkage, which results in increased stresses in the surface layers of the material. When the second polymer layer is applied to the first, similar processes occur, which lead to an increase in surface stresses in the first layer. If the forces of surface stresses in the first layer exceed the forces of interfacial adhesion of the polymer to the table material, then the first layer will detach from the table surface. This leads to the curvature of the lower layers of the product, that is, to the appearance of the warping effect shown in Figure 4. With a further increase in the number of printed layers of the product, the stresses from the curvature of the first layer decrease, the warping effect decreases and the layers are aligned. 


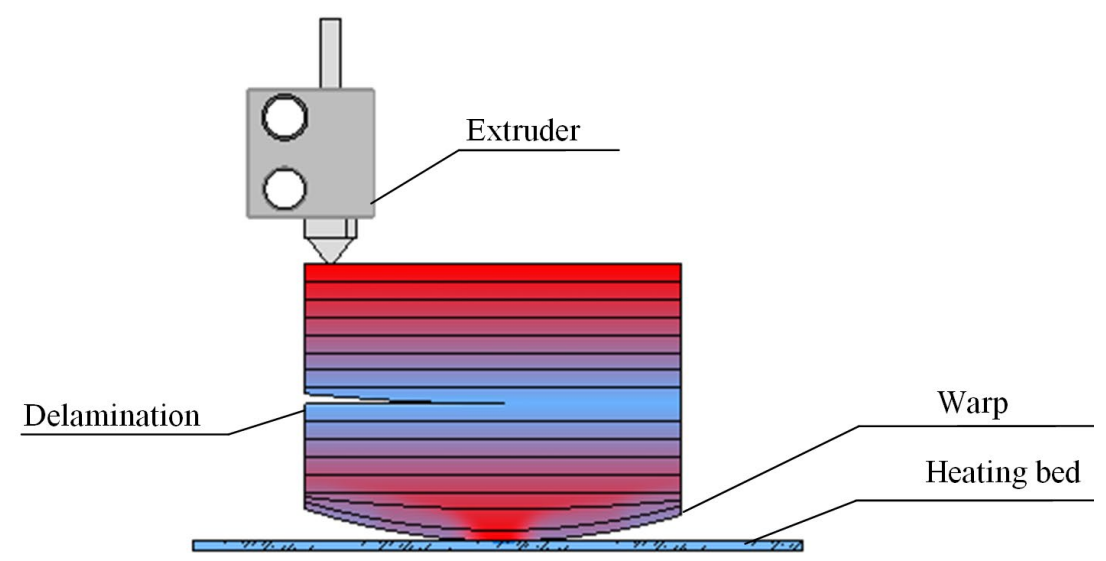

Fig. 4. Effects of warping and delamination of the product.

The high degree of polymer shrinkage rate, at which increased stresses arise between the layers of the product, affects not only the appearance of the warping effect. If surface stress forces appear in the product between the polymer layers, which exceed the interfacial adhesion forces of these layers, then the delamination effect will be observed, that is, the layers will detach (detach) from each other. Figure 4 schematically shows the effect of delamination on the surface of a product. Figure 5 shows the delamination effect of a PEEK polymer product.

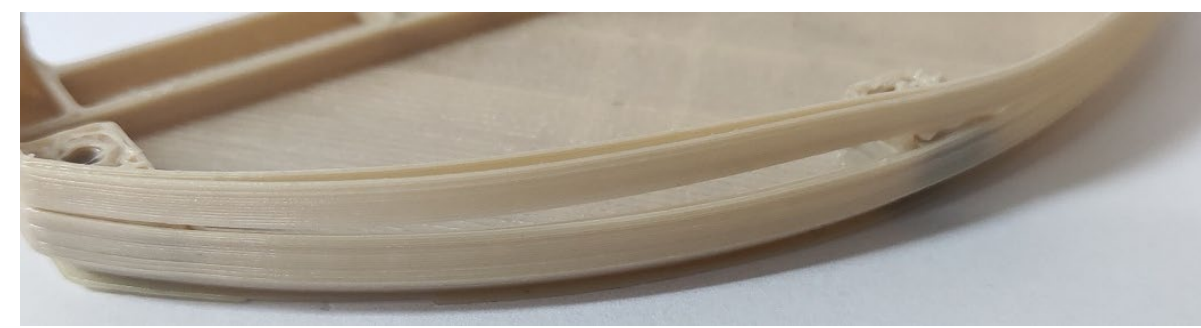

Fig. 5. The delamination effect of a PEEK polymer product

\section{Annealing of polymer products}

When the polymer is extruded into an environment below the glass transition temperature ( $\mathrm{Tg})$, there is a large temperature difference between the melt and the environment, resulting in a high cooling rate of the polymer.

The high polymerization rate is accompanied by increased shrinkage, causing increased internal stresses between the layers. This leads to the appearance of the effects of warping and delamination and, as a consequence, to significant deviations of the geometric dimensions from the given shape of the product.

Figure 6 shows the main characteristics of the printing processes of high-performance polymers at temperatures below the glass transition temperature. 


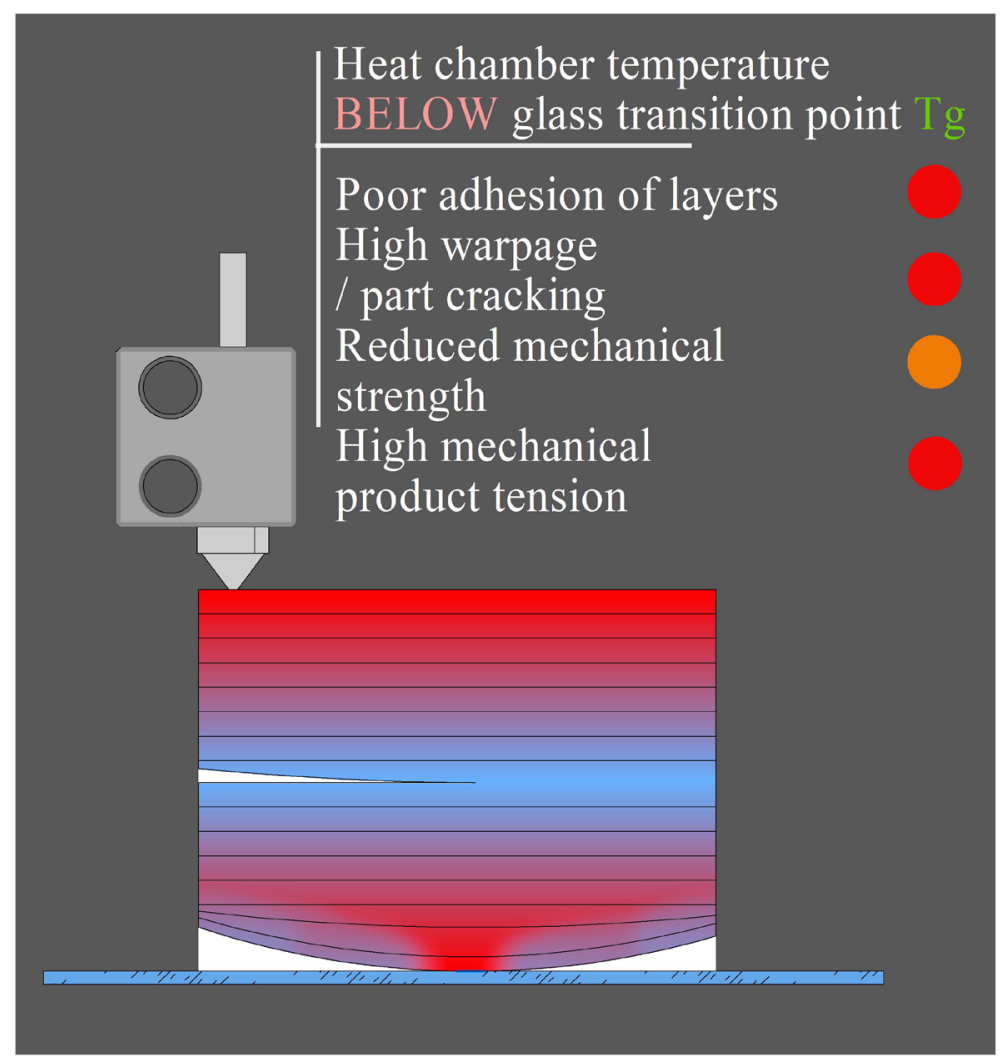

Fig. 6. Characteristic of the printing process below the glass transition temperature.

When printing with high-performance polymers at ambient temperatures below the glass transition temperature, parts are not completely crystallized. An example of such a PEEK polymer print is shown in Figure 7.

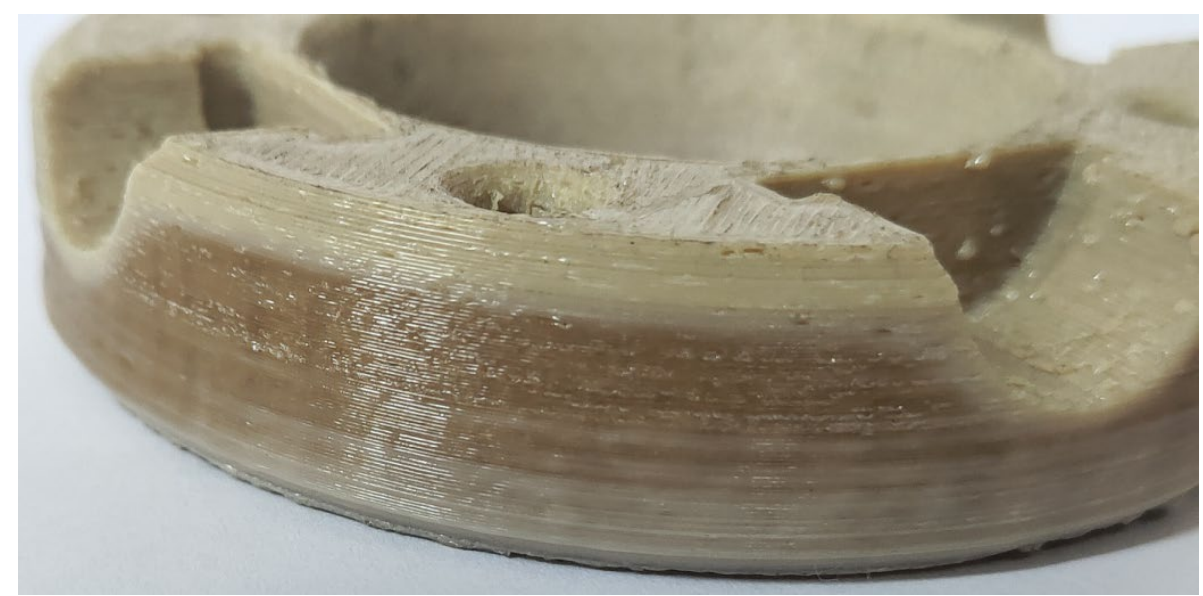

Fig. 7. Sample of PEEK polymer 3D printing at ambient temperature below glass transition temperature.

To improve the quality of products printed by the method described above, thermal annealing is used. 
Annealing is a special heat treatment process that optimizes the molecular structure of the polymer and reduces the interlayer stresses that occur during rapid polymerization.

This process is carried out as follows. The product is placed in an oven, the air temperature is raised above the glass transition temperature of the polymer, and held for a certain period of time. The holding time is calculated depending on the minimum wall thickness of the product. This is followed by slow cooling at a rate of about $10^{\circ} \mathrm{C}$ per hour to ambient temperature.

Figure 8 shows the temperature changing of time during the thermal cycle of annealing PEEK polymer parts [7].

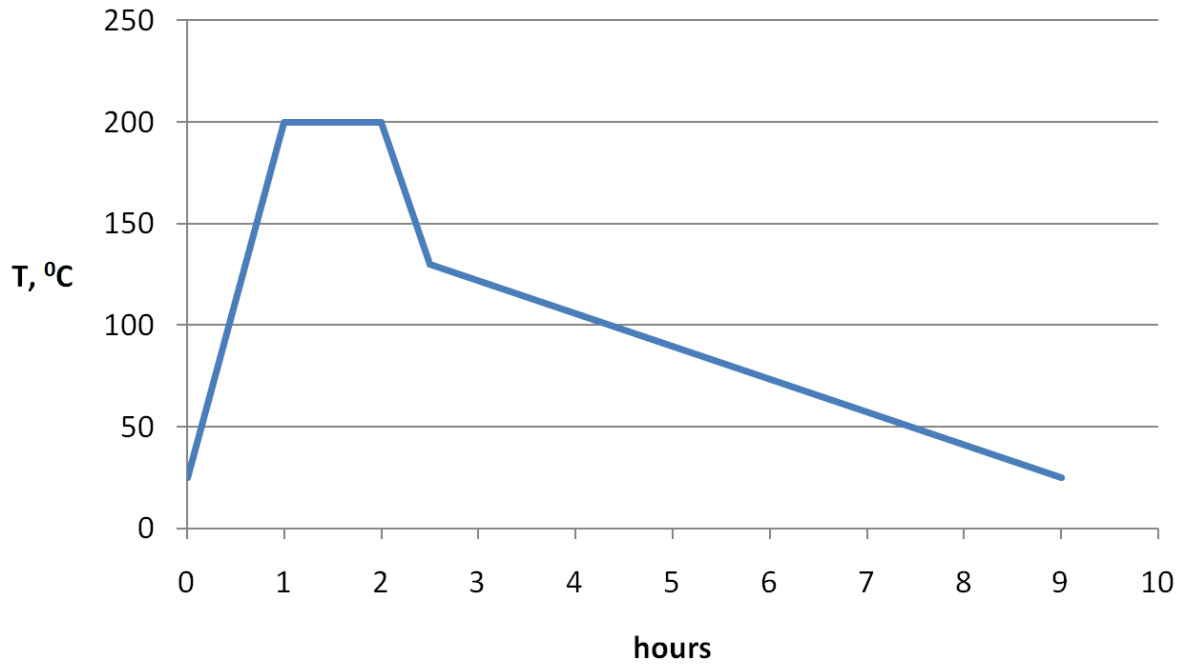

Fig. 8. Annealing thermal cycle for PEEK parts.

When using thermal annealing, some disadvantages of this process must be taken into account: - annealing takes quite a long time and can last more than eight hours, which is not always economically feasible, especially under conditions of mass production;

- in the process of annealing, some change in the size of the product occurs, caused by the rearrangement of polymer grains at the microscopic level;

- heterogeneity in the crystal structure of the product in the presence of walls of different thickness. Thicker walls remain unannealed;

- using thermal annealing, it is impossible to eliminate the distortion of the product shape caused by the effects of warping and delamination.

\section{Heated chamber and what is it for}

The use of a heated chamber in the printing process allows you to change the ambient temperature in which the polymer is cooled. That is, in the heated chamber, it is possible to create favorable conditions for the desired cooling rate, depending on the properties of the polymer to be printed. Providing a controlled polymerization process, allows you to control the degree of polymer shrinkage, leads to a decrease in interlayer stresses and the elimination of the causes of the effects of warpage and delamination. In this case, the process of polymer crystallization occurs with the formation of regular crystal lattices, which provides a more uniform structure of the material and the product does not require further thermal annealing.

When comparing the results of printing products from high-performance polymers made without using a heated chamber and with a heated chamber, it is observed that the product made 
in a heated chamber minimizes deviations of geometric dimensions from a given shape, reduces surface roughness, and products have improved thermal and mechanical properties.

To solve the problems associated with the negative effects of a high degree of shrinkage of high-performance polymers, the research department of VOLTECHNO LLC has developed a heated chamber (Fig. 9), which is used in the design of 3DLIFE THERMO printers. The insulated heated chamber allows you to maintain a constant air temperature up to $250^{\circ} \mathrm{C}$. This makes it possible to provide a controlled printing process with high-performance polymers with good repeatability of the geometric shapes of the finished products. The air is heated to the required temperature by heaters located inside the chamber. Uniform heating of the entire volume of the chamber is carried out using a fan built into the chamber, providing convective heat exchange between the heating elements and the air of the chamber.

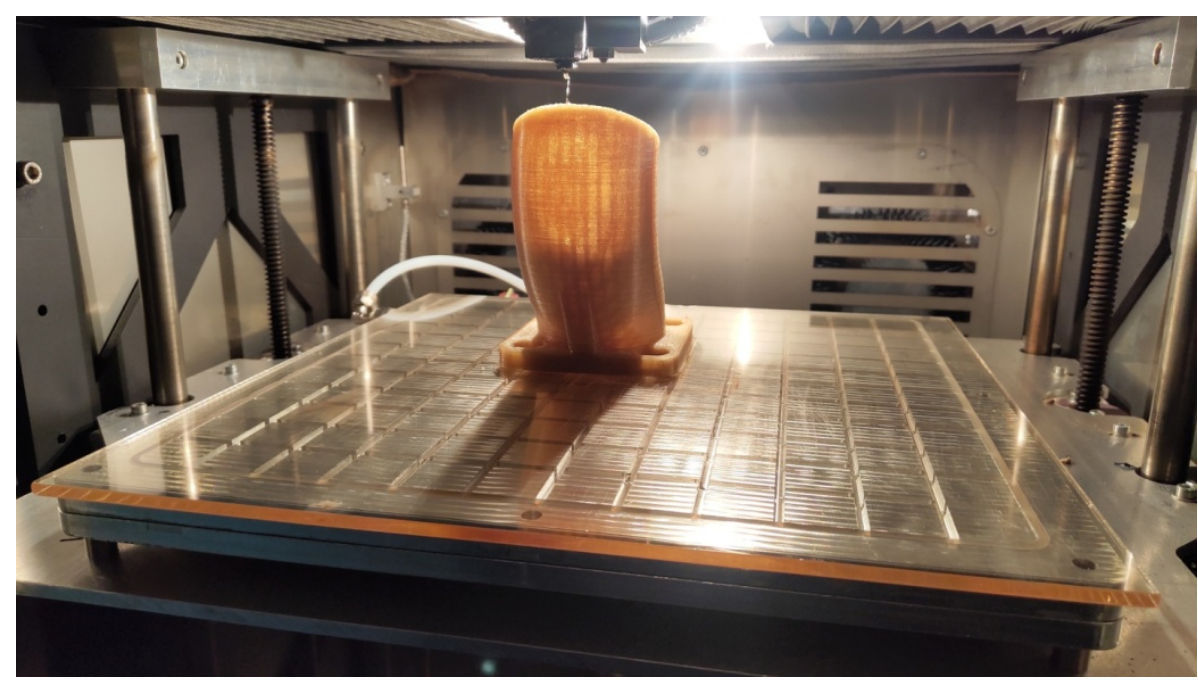

Fig. 9. Heated chamber of the 3DLIFE THERMO printer

It has been found that the optimum ambient temperature maintained in a heated chamber is the glass transition temperature (Tg) of the polymer used.

The $\mathrm{Tg}$ parameter is one of the most important thermophysical properties of amorphous polymers. At this temperature, the polymer chains are randomly oriented and can move freely, while the polymer is in structural relaxation and cools smoothly and uniformly. Thus, the strength and dimensional accuracy of 3D printed high performance polymer parts is achieved [8]. Optimum chamber temperatures for some high performance polymers: PPSU - $225^{\circ} \mathrm{C}$; PEI 1010 - $220^{\circ} \mathrm{C}$; PEKK / PEEK $-160^{\circ} \mathrm{C}$.

Figure 10 shows the main characteristics of the printing processes of high-performance polymers at temperatures above the glass transition temperature. 


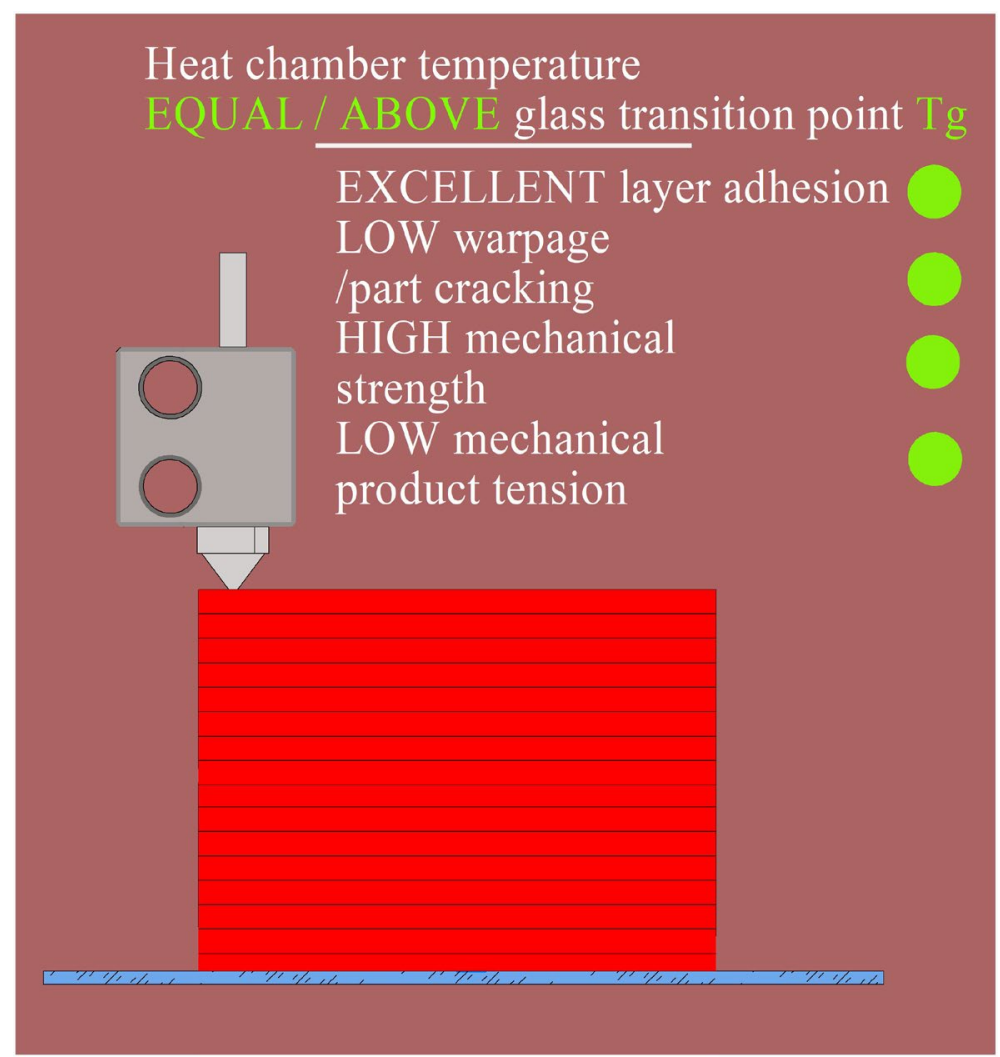

Fig. 10. Characteristic of the $3 D$ printing process above the glass transition temperature

When printing with high-performance polymers at an ambient temperature above the glass transition temperature, the parts are completely crystallized. An example 3D print from PEEK is shown in Figure 11.

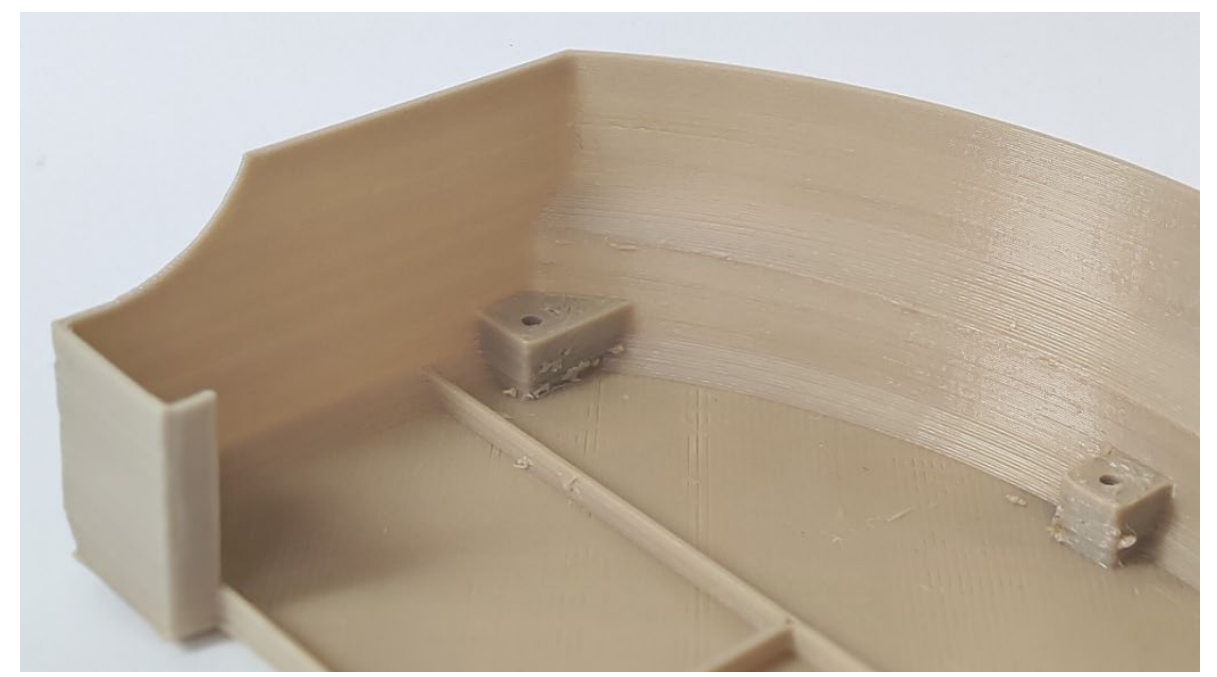

Fig. 11. Sample of PEEK polymer 3D printing at ambient temperature above glass transition temperature

\section{Summary}

The main advantages of the heated chamber: 
1) a high degree of crystallinity of the product is achieved, which leads to improved chemical, mechanical and thermal characteristics;

2) the uniformity of the crystalline structure of the material is ensured, this leads to the same properties throughout the entire volume of the product and eliminates the negative effects of warping and delamination;

3) there is no need for additional heat treatment processes, such as annealing, which leads to a reduction in the production time of finished products;

4) there is no limitation on the minimum wall thickness due to the lack of heat treatment after printing the product;

5) no deformation due to heat treatment, resulting in better shape tolerances and dimensional accuracy.

\section{References}

[1] PEEK: Ensinger extends its hollow rod portfolio.

https://www.ensingerplastics.com/en/press-and-news/press-releases/peek-ensinger-extends-itshollow-rod-portfolio

[2] https://visionminer.com/blogs/articles/carbon-fiber-3d-printing

[3] K. Friedrich, Polymer composites for tribological applications. Institute for Composite Materials (IVW GmbH), Technical University Kaiserslautern, 67663 Kaiserslautern, Erwin Schroedinger Str. 58, Germany. / Advanced Industrial and Engineering Polymer Research 1 (2018), p. 3-39.

https://www.sciencedirect.com/science/article/pii/S2542504818300083?via\%3Dihub

[4] Polyketone: Compounds to meet mechanical, tribological and chemical demands.

https:/www.ensingerplastics.com/en/press-and-news/press-releases/polyketone-compounds-tomeet-mechanical-tribological-and-chemical-demands

[5] E.P. Boytsov, S.I. Blaginin, A.V. Sarazov, A.V. Sinkov, R.N. Titov. Russian Federation patent for useful model No. 189218 "Printing unit for 3D printing with high-temperature polymers using FDM technology" dated 28.01.2019.

[6] A Sviridov, Iu Lopatina, Iu Kurganova. 3D-printed polyether ether ketone samples mechanical properties estimation. Modern Engineering, IOP Publishing, IOP Conf. Series: Materials Science and Engineering 589 (2019) 012021. https://doi.org/10.1088/1757$899 X / 589 / 1 / 012021$

[7] https://www.roboze.com/en/resources/peek-3d-printing-what-does-a-3d-printer-need-toprint-peek.html

[8] https://polymerdatabase.com/polymer\%20physics/GlassTransition.html 